for young women over 19 years of age who have aged out of the Vaccines for Children entitlement program. The objectives of this study were to assess a sample of minority women who attend neighbourhood health centers to determine predictors of vaccination, and to assess for the presence of HPV infection in these women. Those without evidence of prior HPV infection may benefit from a prophylactic vaccine.

Methods Between April 2009 and April 2010, we enrolled a convenience sample of 100 African American and 100 Latina women who completed a computer-assisted personal interview. Participants were queried regarding: demographics, risk for sexually transmitted infections including drug or alcohol use, HPV vaccine willingness, knowledge, attitudes and beliefs, and vaccination status. Frequencies were calculated using SAS, version 9.2. Self-collected vaginal swab samples from 118 participants were tested for HPV using line probe assay.

Results Participants were poor with 113 (57\%) having a household income of $<\$ 20000$; and at risk for HPV infection. One hundred twenty-one (61\%) did not use condom at last sex. However, only 17/ 118 (14\%) were positive for any HPV. Predictors of vaccination could not be determined because there was not sufficient outcome response variation. A vast majority 161 (80\%) of participants had not received HPV vaccination, though a most 136 (68\%) reported willingness.

Conclusions Three years after vaccine approval, the majority in a sample of vulnerable women had not been vaccinated despite their willingness. Public health campaigns have been successful at raising awareness and making vaccine acceptable, but may be less successful at providing the vaccine to vulnerable women. Strategies should focus on delivering vaccine to African American and Latina women in order to decrease cervical cancer disparities.

\section{S14.3 EVALUATING THE INTERNET AS AN STD RISK ENVIRONMENT FOR TEENS: FINDINGS FROM THE COMMUNICATION, HEALTH, AND TEENS (CH@T) STUDY}

doi:10.1136/sextrans-2011-050102.58

${ }^{1} \mathrm{E}$ R Buhi, ${ }^{1} \mathrm{~N}$ Klinkenberger, ${ }^{1} \mathrm{H}$ Blunt, ${ }^{1} \mathrm{E} \mathrm{M}$ Daley, ${ }^{1} \mathrm{~J}$ Baldwin, ${ }^{2} \mathrm{C}$ Rietmeijer. ${ }^{1}$ University of South Florida, College of Public Health, Tampa, Florida, USA; ${ }^{2}$ Colorado School of Public Health, Denver, Colorado, USA

Background The CH@T study aims to identify/characterise a group of teens reporting meeting sex partners online and a group reporting meeting sex partners in-person (not online) and examine the differences between the 2 groups in sexual health risks.

Methods Youth aged $13-19$ years $(\mathrm{N}=273)$ visiting a publiclyfunded clinic completed a 20-min Audio-Computer Assisted SelfInterview. Included were global sexual behaviour questions (eg, oral/ vaginal/anal sex experience, number of sex partners) and specific partnership history questions, including meeting partners online/ offline. Participants were also tested for chlamydia/gonorrhea. Audio-Computer Assisted Self-Interview responses were anonymously linked to teens' biological STD results. A $\chi^{2}$ test was performed to determine the association between meeting a sex partner online and current STD status.

Results Participants identified as female (89.4\%) and heterosexual $(80.7 \%)$. Nearly 9 in 10 (88.6\%) reported oral, 97.8\% vaginal, and $28.6 \%$ anal sex experience. Of those with biological STD data $(n=267), 14.2 \%$ had a current STD infection. Of all teens, $15.4 \%$ $(n=42)$ reported having sex with a partner originally met online (of these, $>$ half $[57.1 \%]$ met $>1$ partner). Compared with teens reporting only partners met in-person, teens reporting an online partner had significantly greater numbers of oral, vaginal, and anal sex partners. However, analyses indicated no association between having a current STD and reporting an online partner, $\chi^{2}(1, N=267)=0.95, p=0.34$.

Conclusions Theory-driven STD prevention and sexual health promotion interventions should be tailored to meet specific needs of young people seeking partners both online and offline. Sex-seeking, dating, and social networking websites may represent important intervention contexts.

\section{S14.4 DETECTING CHLAMYDIAL AND GONOCOCCAL INFECTIONS THROUGH SOCIAL AND SEXUAL NETWORKS}

doi:10.1136/sextrans-2011-050102.59

${ }^{1,2} \mathrm{~A}$ A Al-Tayyib, ${ }^{1,2} \mathrm{C}$ A Rietmeijer. ${ }^{1}$ Denver Public Health, Denver, Colorado, USA; ${ }^{2}$ Colorado School of Public Health, Denver, Colorado, USA

Background The overall goal of this ASTDA award was to adapt and evaluate Respondent-Driven Sampling (RDS) to enhance Chlamydia trachomatis (Ct) and Neisseria gonorrhoeae (GC) screening activities. Specifically, the aims were to develop and evaluate a programmatic approach using RDS as a method to prospectively screen for $\mathrm{Ct} / \mathrm{GC}$ in networks of infected persons, to compare the prevalence of $\mathrm{Ct} /$ GC infections detected via current venue-based screening activities to the prevalence of infection detected using RDS as a referral mechanism, and to determine and compare the cost per infection detected via current screening activities to the cost per infection using RDS.

Methods Using two components of RDS, the systematic referral scheme and the dual incentive structure, we developed a program to refer social and sexual contacts for $\mathrm{Ct} / \mathrm{GC}$ screening. Initial seed participants ages 15-24 were identified and asked to recruit their peers for screening, who in turn recruited their peers, and so on. Persons received incentive for their own participation $(\$ 10)$ in addition to incentive for participation of those they refer $(\$ 10)$. Participants could refer up to 5 social and sexual contacts. Participants provided a urine specimen for $\mathrm{Ct} / \mathrm{GC}$ screening and completed a brief survey questionnaire.

Results Between December 2008 and March 2010, 66 initial seeds were recruited. Of the 66 seeds, $17(25 \%)$ were successful in recruiting referrals. A total of 439 referrals were recruited, resulting in 7 networks initiated from an infected seed and 10 networks from a non-infected seed. The majority of referrals, 372 (85\%), belonged to a single network (Abstract S14.4 figure 1). The remaining 67 referrals belonged to 16 networks ranging in size from 2 to 18 members. Across all networks, $67 \%$ of referrals were male and $33 \%$ were female. The overall prevalence of infection was $5.7 \%$ for $\mathrm{Ct}$ and $6.9 \%$ for GC among the referrals. This is compared to a prevalence of $12.2 \%$ for $\mathrm{Ct}$ and $1.5 \%$ for GC detected through venue-based screening in the same age demographic.

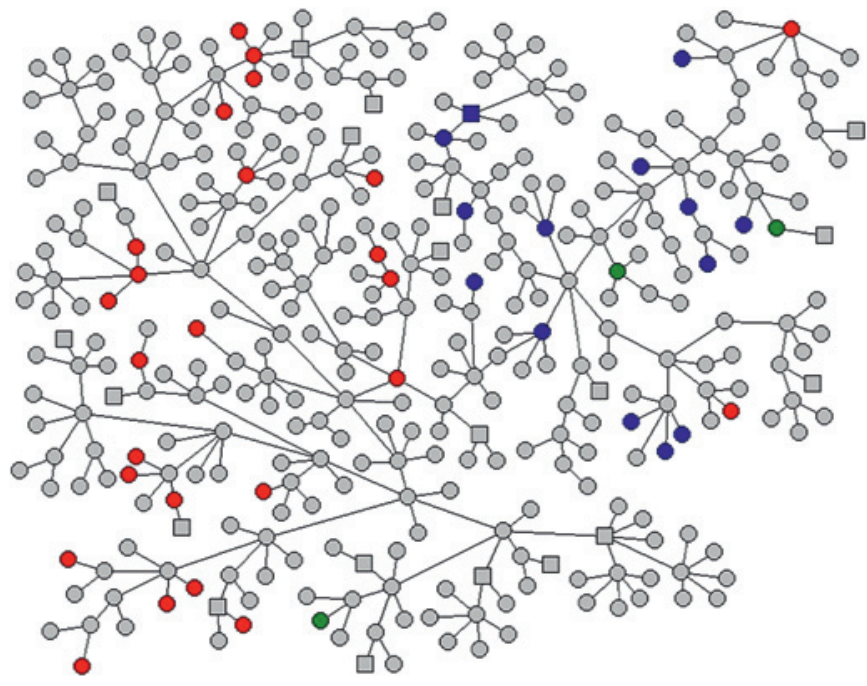

Abstract S14.4 Figure 1 